Polymer Journal, Vol. 9, No. 1, pp 41-45 (1977)

\title{
Laser Flash Photolysis Studies of Copolymers of Phenyl Vinyl Ketone and Vinylnaphthalene in Benzene Solution
}

\author{
Koichiro Hayashi, ${ }^{*}$ Masahiro IRIE, ${ }^{*}$ John KIwI, ${ }^{* *}$ and Wolfram Schnabel ${ }^{* *}$ \\ *Institute of Scientific and Industrial Research, Osaka University, Osaka, Japan. \\ **Hahn-Meitner-Institut für Kernforschung Berlin GmbH, \\ Bereich Strahlenchemie, D 1000 Berlin 39.
}

(Received July 30, 1976)

\begin{abstract}
Yields and lifetimes of triplet states of 2-vinylnaphthalene (2-VN) incorporated as comonomer in copolymers with phenyl vinyl ketone (PVK) were measured in benzene solution at room temperature. Samples were irradiated with 347.1-nm light flashes. Light absorbed by PVK moieties was effectively transferred to 2-VN moieties. With a copolymer sample containing about 20-mol\% 2-VN and possessing at the end of the flash about 20 triplet excited 2-VN base units per macromolecule, it was possible to evidence both inter- and intramolecular $\mathrm{T}-\mathrm{T}$ annihilation.

The triplet energy transfer from PVK to 2-VN appeared to be accomplished predominantly by an intramolecular process possibly-including energy migration along the chain within PVK segments.
\end{abstract}

KEY WORDS Laser Flash Photolysis / Copolymer of Phenyl Vinyl

Ketone and Vinylnaphthalene / Poly(2-vinylnaphthalene) / T-T

Annihilation / Triplet Energy Transfer / Quantum Yield / Exciton /

We reported recently on transient absorption spectra of poly(phenyl vinyl ketone) $\mathrm{PPVK}^{1}$ and copolymers of phenyl vinyl ketone with methyl methacrylate MMA and other comonomers ${ }^{2}$ obtained by irradiating polymer solutions with 25-nsec laser light flashes $(\lambda=347.1 \mathrm{~nm})$. The transient absorptions were assigned to PVK base units excited in their triplet states. It was found that the lifetime of excited PVK base units located as isolated units in MMA chains was relatively long (several $\mu \mathrm{sec}$ ). Thus it was possible to investigate conveniently the processes involved in the triplet photosensitization ${ }^{3}$ as well as in the quenching in polymer systems, such as intramolecular selfquenching or triplet-triplet annihilation, by use of a laser. The present work also is aimed at obtaining information along this line. Copolymers of PVK and vinylnaphthalene, which is an effective photosensitizer for the isomerization reaction of stilbene, ${ }^{3}$ were considered as appropriate materials for this purpose for the following reasons:

(a) Naphthalene is known to quench triplet excited PPVK, whereby the formation of the absorption spectrum of triplet naphthalene is sensitized. ${ }^{1,4}$ The lifetime of triplet naphthalene thus formed is relatively long (several $\mu \mathrm{sec}$ ) and enables one to easily follow its decay. It was assumed that moieties with pendent naphthalene groups incorporated in a PPVK chain should behave analogously. ${ }^{3}$

(b) Naphthalene does not absorb light at $\lambda=$ $347.1 \mathrm{~nm}$. Therefore, any naphthalene triplet absorption detectable should originate from PVK-triplets having excitation energy transferred to naphthalene.

Based on the fact that photolyzing light is exclusively absorbed by PVK base units, the following reaction scheme is postulated, ${ }^{5}$ where ${ }^{1} \mathrm{PVK},{ }^{3} \mathrm{PVK}^{*},{ }^{1} \mathrm{VN}$, and ${ }^{3} \mathrm{VN}^{*}$ designate ground and excited triplet states of PVK and VN base units in the copolymer:

$$
\begin{gathered}
{ }^{1} \mathrm{PVK}^{\stackrel{h_{\nu}}{\longrightarrow}}{ }^{3} \mathrm{PVK}^{*} \\
{ }^{3} \mathrm{PVK}^{*} \stackrel{k_{2}}{\longrightarrow}{ }^{1} \mathrm{PVK} \\
{ }^{3} \mathrm{PVK}^{*}+{ }^{1} \mathrm{VN} \stackrel{k_{3}}{\longrightarrow}{ }^{1} \mathrm{PVK}+{ }^{3} \mathrm{VN}^{*} \\
{ }^{3} \mathrm{VN}^{*} \stackrel{k_{4}}{\longrightarrow} \mathrm{VN}^{1} \\
{ }^{3} \mathrm{VN}^{*}+{ }^{3} \mathrm{VN}^{*} \stackrel{k_{5}}{\longrightarrow} \mathrm{VN}^{*}+{ }^{1} \mathrm{VN}
\end{gathered}
$$




\section{EXPERIMENTAL}

Phenyl vinyl ketone (PVK) was prepared by Hofmann degradation of $\beta$-dimethylaminopropiophenone hydrochloride. 2-Vinylnaphthalene (2VN) was recrystallized from ethanol solution. Appropriate monomer mixtures were polymerized in benzene solution at $60^{\circ} \mathrm{C}$ using $\alpha, \alpha^{\prime}$-azobisisobutylonitrile as an initiator. The conversion was less than $10 \%$. The copolymers were reprecipitated several times from benzene solution with methanol and dried in vacuo. The copolymer composition was determined by elemental analysis. Five copolymer samples were used, with 2-VN contents of $2.8,7.8,11.8,16.4$, and $19.7 \mathrm{~mol} \%$. The number average molecular weight of the samples determined by membrane osmometry in toluene varied between 2 and $7 \times 10^{5}$.

The copolymers were dissolved in benzene. Dilute solutions were irradiated in rectangular cells and were freed from air by bubbling with purified Ar. A ruby laser (Korad Model K1QS2) was used as the photolyzing light source $(\lambda=$ $347.1 \mathrm{~nm}$ ) and a Xenon lamp (Osram XBO $450 \mathrm{~W}$ ) as the analyzing light source. The intensity of the incident light was reduced by Schott BG 23 filters. Further details have been reported before. $^{1,2}$

\section{RESULTS AND DISCUSSION}

\section{Absorption Spectra and Lifetime of Transient Species}

Both absorption and emission were observed in the wavelength range of about 320 to $600 \mathrm{~nm}$.

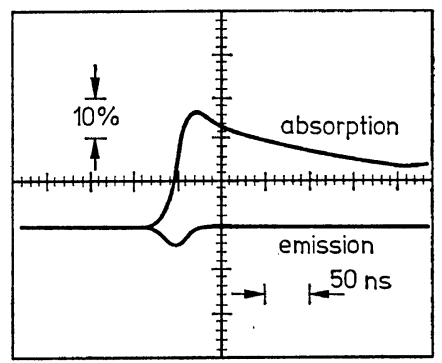

Figure 1. Oscilloscope trace obtained at $\lambda=430 \mathrm{~nm}$ with poly(PVK-co-2-VN) containing 2.8-mol\% 2VN in benzene solution: Copolymer concentration, $2.1 \times 10^{-3}$ base $\mathrm{mol} / l$; absorbed dose, $c a .1 .5 \times 10^{-4}$ einstein $/ l$.

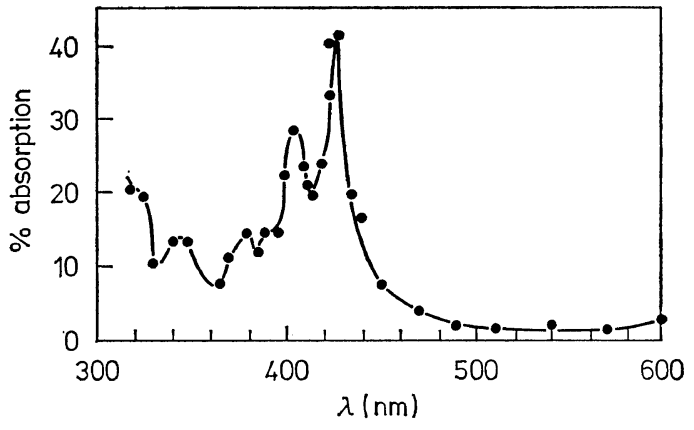

Figure 2. Absorption spectrum observed at the end of the flash in benzene solution containing poly(PVK-co-2-VN): 2-VN fraction, $2.8 \mathrm{~mol} \%$; copolymer concentration, $2.1 \times 10^{-3}$ base $\mathrm{mol} / \mathrm{l}$.

A typical oscilloscope trace is shown in Figure 1. The emission decayed within the duration of the flash. The absorption was formed rapidly within the flash. The absorption spectrum observed immediately at the end of the flash is shown in Figure 2. It is similar to the $\mathbf{T}-\mathbf{T}$ absorption spectrum of naphthalene reported by other authors. $^{6-8}$ However, the peak at $\lambda=413 \mathrm{~nm}$ observed with naphthalene is shifted to about $430 \mathrm{~nm}$. In the range of 400 to $410 \mathrm{~nm}$ the copolymer exhibits a minor peak. The decay kinetics were independent of wavelength, which allows the conclusion that the observed absorption spectrum is due to a single transient species. The decay of the spectrum was accelerated in the presence of oxygen and stilbene. Therefore the spectrum was assigned to the triplet state of excited 2-VN base units formed by energy transfer from PVK to 2-VN units.

For several reasons it is believed that the optical absorption of PVK triplets does not interfere with the observation of naphthalene triplets:

(i) A homo PPVK sample irradiated with a 347.1-nm light flash under the same conditions as used for copolymer samples containing VN yielded an optical density at $\lambda=430 \mathrm{~nm}$ about 60 times less than observed, e.g., with a copolymer sample containing $2.8-\mathrm{mol} \% 2 \mathrm{VN}$.

(ii) The half-life of PPVK triplets amounts to about 70 nsec. $^{1}$ It was not found in this work that a portion of the optical density decayed with this rate, as would be assumed if the observed spectrum would be constituted by an 
addition of the absorptions of both PVK and 2-VN triplets.

(iii) The formation of the absorption during the flash appeared to occur very rapidly, thus indicating a very effective energy transfer from PVK triplet states to 2-VN.

It was found that the decay behavior of the absorption spectrum shown in Figure 2 was influenced remarkably by the intensity of the incident light, as demonstrated in Figure 4. The extent of the absorption, furthermore, depends upon the copolymer composition, as demonstrated in Figure 6. Both effects will be discussed in detail below.

\section{Influence of Intensity of Photolyzing Light on the} Decay Rate

As shown in Figure 3 the absorption depends linearly on the absorbed dose per flash, indicating that the triplet concentration at the end of the flash is proportional to intensity.

The significant influence of the intensity of the incident light on the decay of the transient absorption is shown in Figure 4, where the optical density at $\lambda=430 \mathrm{~nm}$ is plotted semilogarithmically $v s$. time. It is seen that the plots exhibit initial curvatures, if the absorbed dose per flash is greater than about $2 \times 10^{-5}$ einstein/l.

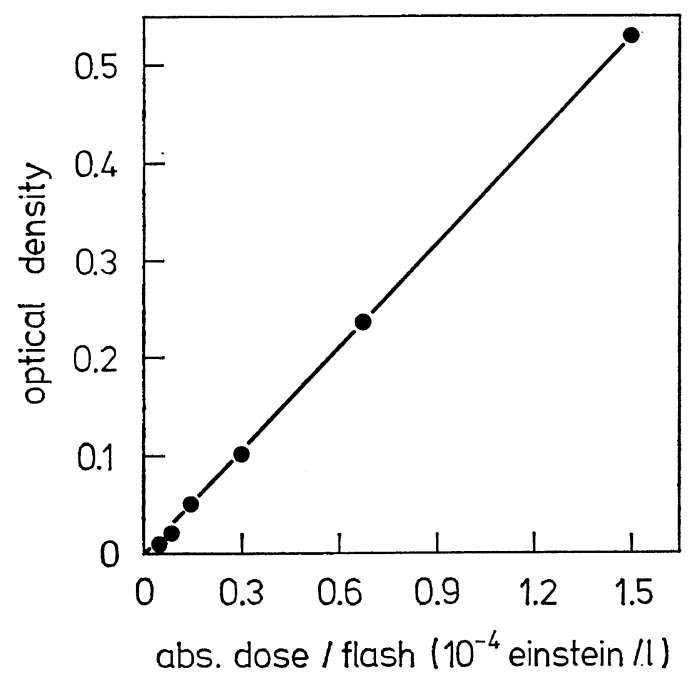

Figure 3. Optical density at $\lambda=430 \mathrm{~nm} v s$. the absorbed dose per flash: Half width of flash, 25 nsec; poly(PVK-co-2-VN) in benzene solution $\left(2.25 \times 10^{-3}\right.$ base $\left.\mathrm{mol} / \mathrm{l}\right) ; 2-\mathrm{VN}$ fraction in copolymer, 0.197 .

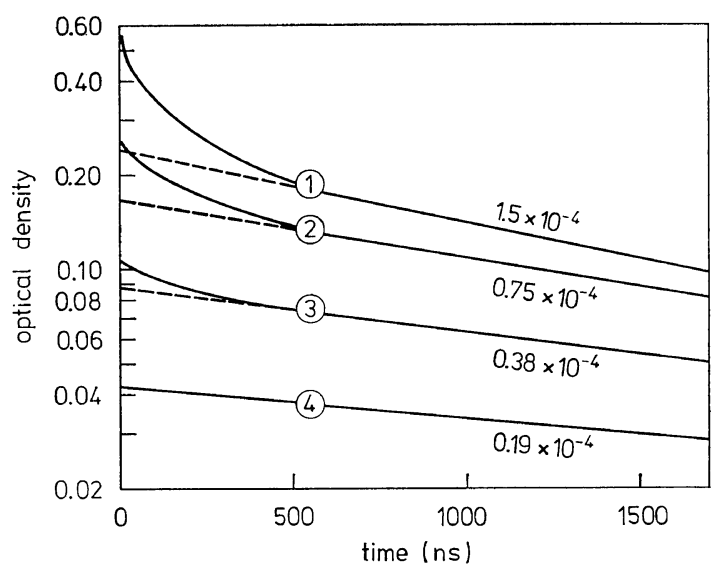

Figure 4. Semilogarithmic plot of the optical density at $\lambda=430 \mathrm{~nm}$ vs. time: Poly(PVK-co-2-VN) containing $19.7-\mathrm{mol} \% 2-\mathrm{VN}$; copolymer concentration, $2.25 \times 10^{-3}$ base $\mathrm{mol} / \mathrm{l}$. The numbers at the curves indicate the absorbed dose per flash in (einstein/l).

At later times the decay follows apparently first-order kinetics. The slopes of the linear portions of the curves depend also on the intensity of the incident light, which suggests that intermolecular triplet-triplet annihilation is occurring. The rate constant of the latter process $k_{5}$ (inter) was evaluated in the following way. By plotting the first-order rate constant $k_{\mathrm{obs}}$ obtained from the linear portions of the curves in Figure $4 v s$. the concentration of 2-VN triplets $\left[{ }^{3} \mathrm{VN}^{*}\right]$, a straight line was obtained, as shown in Figure 5. Thus, it can be assumed that formally the following relationship holds:

$$
k_{\text {obs }}=\sum k_{1}+k_{5} \text { (inter) }\left[\mathrm{VN}^{*}\right]
$$

where $\sum k_{1}$ is the sum of rate constants of firstorder processes occurring simultaneously with intermolecular $\mathrm{T}-\mathrm{T}$ annihilation. Values of triplet concentrations were estimated from O.D. values obtained by extrapolation of the straight line portions in Figure 4 to $t=0$ (as indicated by dotted lines). These values represent the concentration of the isolated triplet in the chain, which will not deactivate by intramolecular triplet-triplet interaction. The extinction coefficient of $2-\mathrm{VN}$ triplets at $\lambda=430 \mathrm{~nm}$ was obtained as $\varepsilon=2.2 \times 10^{4} \mathrm{M}^{-1} \mathrm{~cm}^{-1}$ by using a measured value of the absorbed dose per flash 


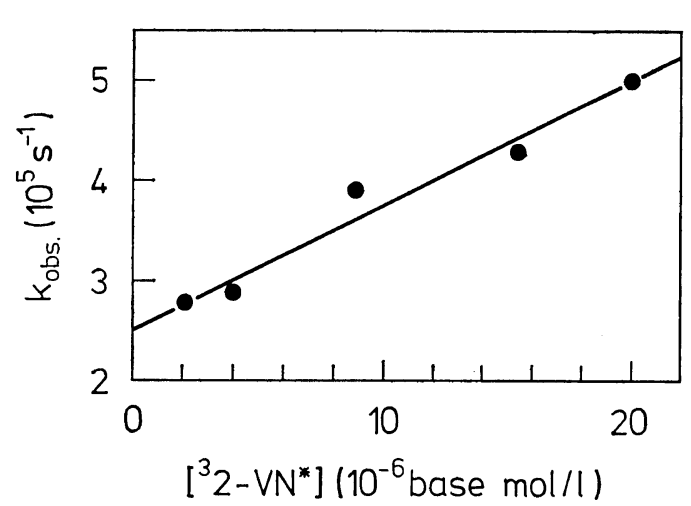

Figure 5. First order rate constant obtained from linear portions of curves in Figure $4 \mathrm{vs}$. the initial concentration of 2-VN-triplets.

of $1.9 \times 10^{-5}$ einstein $/ l$ (curve 4) and an initial O.D. of 0.043 , and assuming quantum yield $\phi$ (triplets $)=0.2$. It agrees fairly well with the value of homogeneously distributed micromolecular naphthalene triplets. ${ }^{6}$ From the slope of the straight line in Figure $5 k_{5}$ (inter) $=1.1 \times$ $10^{10} M^{-1} \mathrm{sec}^{-1}$ is obtained. The rapid decay of the absorption immediately after the flash suggests intramolecular triplet-triplet deactivation.

We estimated that in the experiment represented by curve (1) in Figure 4 on the average there are initially about 20 triplets per macromolecule, whereas the average number of triplets per chain amounts to about 2 to 3 in the experiment pertaining to curve (4) of Figure 4. This estimate explains satisfactorily why in the latter case no indication of intramolecular triplet-triplet annihilation was observed. (It should be noted that the estimate of the number of triplets per chain was based on the assumption that $\phi$ (triplets $)=0.2$ and that all PVK triplets are quantitatively converted to 2-VN triplets.)

Triplet Energy Transfer from PVK Units to 2-VN Units

In principle, process (3) may also occur in the modes of inter- and intramolecular reactions. If energy migration takes place within the PVK sequences of the copolymer chains, intramolecular energy transfer should be an extremely efficient process and in dilute solutions the probability for intermolecular energy transfer should be negligibly low in our case. A series of experiments carried out to elucidate this situation

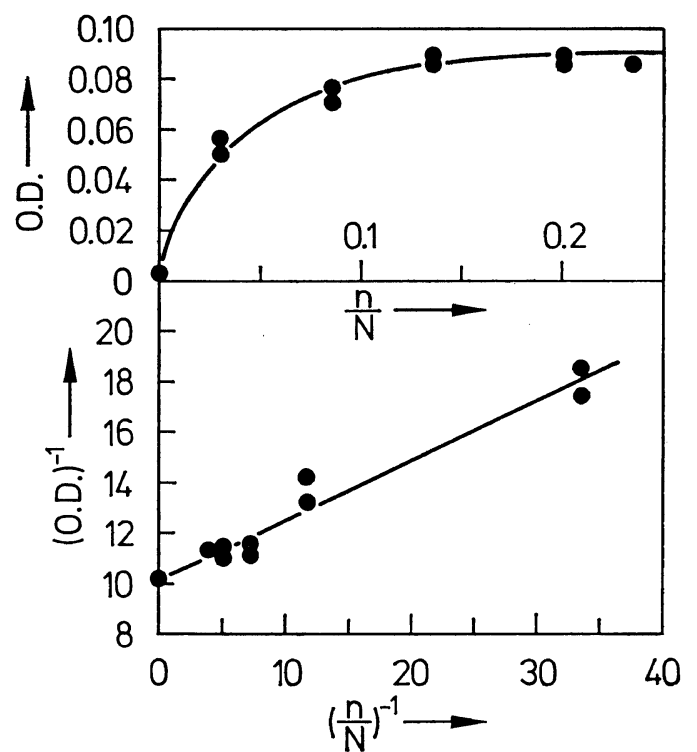

Figure 6. (a) The optical density at $\lambda=430 \mathrm{~nm}$ due to $\mathrm{T}-\mathrm{T}$ absorption of 2-VN-triplets as a function of $2-\mathrm{VN}$ content. The latter was varied by the use of copolymers containing different fractions of 2-VN: [PVK], $1.8 \times 10^{-3}$ base $\mathrm{mol} / l$ at all runs; absorbed dose per flash, $3.8 \times 10^{-5}$ einstein $/ l$. (b) Data of Figure 6(a) plotted according to eq 8. $(n / N$ : fraction of $\mathrm{VN}$ units in copolymer.)

consisted in the following: Copolymer samples containing varying fractions of $2-\mathrm{VN}$ were dissolved in benzene at such concentrations as to keep constant the number of PVK base units per unit volume and to increase the number of 2-VN base units per unit volume. Thus the absorbed dose per flash remained constant (about $3.8 \times 10^{-5}$ einstein $\left./ l\right)$. As is shown in Figure 6(a), the optical density measured at the end of the flash increases steadily with increasing 2-VN content and approaches a limiting value at higher 2-VN content, which indicates that at higher 2VN contents all PVK triplets are converted to naphthalene triplets.

Following the concept of energy migration along the chain via a process analogous to the exciton hopping mechanism in crystals of low molecular weight compounds, ${ }^{9}$ we tried to estimate the exciton jump frequency from the data presented in Figure 6(a). In order to apply the formal treatment of the exciton hopping model to our system, it is necessary to assume (a) that the vinylnaphthalene base units are randomly 
distributed along the macromolecule and (b) that a macromolecule can be considered as a quasiunidimensional system, i.e., that intramolecular energy migration involves exclusively proximate base units and does not occur via jumps between base units of different segments of the same molecule. By assuming that our quasi-unidimensional system may be treated in a way analogous to three-dimensional systems, which the exciton hopping model is normally applied for, we arrived at the following expressions: The fraction of PVK triplets converted to naphthalene triplets via migration along the chain is

$$
\frac{\phi\left({ }^{3} \mathrm{VN}^{*}\right)}{\phi\left({ }^{3} \mathrm{PVK}^{*}\right)}=\frac{\nu(n / N)}{k_{2}+\nu(n / N)}
$$

Equation 7 can be transformed to

$$
\frac{1}{\phi\left({ }^{3} \mathrm{VN}^{*}\right)}=\frac{1}{\phi\left({ }^{3} \mathrm{PVK}^{*}\right)}\left\{1+\frac{k_{2} N}{\nu n}\right\}
$$

$\phi\left({ }^{3} \mathrm{VN}^{*}\right)$, quantum yield of naphthalene triplets

$\phi\left({ }^{3} \mathrm{PVK}^{*}\right)$, quantum yield of PVK triplets formed initially

$n$, average number of $\mathrm{VN}$ units per chain

$N$, average number of base units per chain

$\nu$, mean exciton jump frequency

As shown in Figure 6(b), a plot of the reciprocal optical density due to the absorption of naphthalene triplets $v s .(n / N)^{-1}$ yields a straight line, as expected from eq 8 . With $k_{2}=1 \times 10^{-7}$ $\mathrm{sec}^{-1}, 1$ one obtains from the slope of the straight line in Figure $6(\mathrm{~b}) \nu=4 \times 10^{8} \mathrm{sec}^{-1}$. It was thus assumed that jumps between PVK units occur with the same frequency as jumps from PVK to VN units. The $\nu$-value obtained is definitely lower than the value reported ${ }^{9,10}$ for crystalline benzophenone $\left(7.5 \times 10^{9} \mathrm{sec}^{-1}\right)$. However, it is significantly higher than the value found for glassy benzophenone $\left(7.5 \times 10^{6} \mathrm{sec}^{-1}\right) .^{9,10}$

The relative position of the $\nu$-value obtained in this work for a polymeric system in between the value of a crystalline low molecular weight compound and the value of its amorphous modification appears to show that the application of the unidimensional mode of the exciton hopping mechanism is feasible for a polymeric system.

\section{REFERENCES}

1. G. Beck, G. Dobrowolski, J. Kiwi, and W. Schnabel, Macromolecules, 8, 9 (1975).

2. J. Kiwi and W. Schnabel, ibid., 8, 430 (1975).

3. S. Irie, M. Irie, Y. Yamamoto, and K. Hayashi, ibid., 8, 424 (1975).

4. a) C. David, W. Demartean, and G. Geuskens, Eur. Polym. J., 6, 1405 (1970).

b) I. Lukač, P. Hradlovič, Z. Maňásak, and D. Belluš, J. Polym. Sci., Part A-1, 9, 69 (1971).

c) A. C. Sommersall and J. E. Guillet, Macromolecules, 6, 218 (1973).

5. T. Takemura, H. Baba, and M. Fujita, Bull. Chem. Soc. Jpn, 46, 2625 (1973).

6. E. J. Land, Proc. Roy. Soc., Ser., A, 305, 457 (1968).

7. G. Proter and M. W. Windsor, ibid., 245, 238 (1958).

8. S. G. Hadley and R. Keller, J. Phys. Chem., 73, 4351 (1969).

9. J. B. Birks, "Photophysics of Aromatic Molecules," Wiley-Interscience, London, 1970, p 548.

10. T. F. Hunter, R. D. McAlpine, and R.M. Hochstrasser, J. Chem. Phys., 50, 1140 (1969). 